BULLETIN OF THE

AMERICAN MATHEMATICAL SOCIETY

Volume 81, Number 1 , January 1975

\title{
ULTRAFILTERS AND ALMOST DISJOINT SETS. II
}

\author{
BY KAREL PRIKRY 1
}

Communicated by W. Wistar Comfort, August 6, 1974

Unless otherwise stated, $\kappa$ is an arbitrary infinite regular cardinal. For every infinite cardinal $\kappa, \mu \kappa$ is the family of uniform ultrafilters on $\kappa$. Our main result is:

THEOREM 1. Suppose that $2^{\kappa}=\kappa^{+}$. Then for every $U \in \mu \kappa$ there is a family $\left\{a_{x}: x \in U\right\}$ such that: for every $x \in U, a_{x} \subseteq x$ and $\left|a_{x}\right|=$ $\kappa$; and for every $x, y \in U$ with $x \neq y,\left|a_{x} \cap a_{y}\right|<\kappa$.

This answers a question of Comfort communicated privately to the author and partially answers a question of Hindman [5]. It is still open whether Theorem 1 holds for singular $\kappa$ as well. The hypothesis $2^{\kappa}=\kappa^{+}$cannot be outright removed, since by [1] it is consistent with ZFC that there is no $A \subseteq P(\kappa)$ such that $|A|=2^{\kappa},|a|=\kappa$ for every $a \in A$, and $|a \cap b|<\kappa$ for every $a, b \in A$ with $a \neq b$. See [4], [5] and [8] for other relevant results.

Definition 1. For $A \subseteq P(\kappa)$ and ideal $I \subseteq P(\kappa), I$ is said to be dense in $A$ modulo sets of power $<\kappa$ if for each $x \in A$ such that $|x|=$ $\kappa$, there is some $y \in I$ with $y \subseteq x$ and $|y|=\kappa$. For brevity we shall write " $I$ is dense in $A \bmod (<\kappa)$ ". $I$ is dense $\bmod (<\kappa)$ if $I$ is dense in $P(\kappa) \bmod (<\kappa)$.

"I is $\lambda$-complete" is defined as in [7].

THEOREM 2. For every $U \in \mu \kappa$ there is a $\kappa$-complete ideal $I \subseteq$ $P(\kappa)-U$ such that $I$ is dense $\bmod (<\kappa)$.

Theorem 1 follows from Theorem 2 by induction on ordinals $<\kappa^{+}$, $a_{x}(x \in U)$ being chosen to belong to $I$. See [8, Theorem 1].

AMS (MOS) subject classifications (1970). Primary 02K25, 54D35.

Key words and phrases. Ultrafilter-uniform, regular, weakly selective, ideal- $\kappa$ complete, dense $\bmod (<\kappa)$, function-almost one-to-one, unbounded $\bmod U$.

${ }^{1}$ Research supported by NSF grant GP-43841. 
Theorem 2 for $\kappa=\omega$ is trivial-let $I=P(\omega)-U$. Thus from now on, $\kappa>\omega$. Theorem 2 follows from Lemma 1 and Lemma 1 clearly follows from Lemmas 2 and 3.

LEMMA 1. If $U \in \mu \kappa$, then there is a $\kappa$-complete ideal $I \subseteq P(\kappa)-U$ which is dense in $U \bmod (<\kappa)$.

To prove Theorem 2, let $I$ be as in Lemma 1 and set $x \in \bar{I}$ iff there are $x_{0}, x_{1}$ such that $x=x_{0} \cup x_{1}, x_{0} \in I$ and there is no $y \in I$ with $y \subseteq x_{1}$ and $|y|=\kappa$. Then $\bar{I}$ is as required.

For $f, g \in{ }^{\kappa}{ }_{\kappa}$ we shall write $f \sim g(\bmod U)$ or $f<g(\bmod U)$ if $\{\rho: f(\rho)=g(\rho)\} \in U$ or $\{\rho: f(\rho)<g(\rho)\} \in U$ respectively.

DEFINITION 2 [2]. $U \in \mu \kappa$ is regular if there is a family $X=$ $\left\{x_{\xi}: \xi \in \kappa\right\} \subset U$ such that for every infinite $S \subseteq \kappa, \cap\left\{x_{\xi}: \xi \in S\right\}=0$.

Definition 3 [6]. $f \in{ }^{\kappa} \kappa$ is almost one-to-one if for each $\rho \in \kappa$, $\left|f^{-1}(\{\rho\})\right|<\kappa$.

Definition 4. $f \in{ }^{\kappa} \kappa$ is bounded $\bmod U$ if for some $\alpha \in \kappa$, $\{\rho \in \kappa: f(\rho)<\alpha\} \in U$. Otherwise $f$ is unbounded $\bmod U$.

DEFINITION 5. $U \in \mu \kappa$ is weakly selective if for every $f \in{ }^{\kappa} \kappa$ which is unbounded $\bmod U$ there is an almost one-to-one $g \in{ }^{\kappa}{ }_{\kappa}$ such that $f \sim g(\bmod U)$.

LEMмA 2. If $U \in \mu \kappa$ is either regular or not weakly selective, then there is a $\kappa$-complete $I \subseteq P(\kappa)-U$ which is dense in $U \bmod (<\kappa)$.

Proof. See [8, Theorems 5 and 11]. In outline, the proofs are as follows. Suppose first that $U$ is regular. Let $X=\left\{x_{\xi}: \xi \in \kappa\right\}$ be as in Definition 2. Set

$$
I=\left\{y \subseteq \kappa: \exists \eta<\kappa \forall \xi\left(\eta<\xi .<\kappa \rightarrow\left|x_{\xi} \cap y\right|<\kappa\right)\right\}
$$

Then $I$ is the desired ideal.

If $U$ is not weakly selective, fix $f \in{ }^{\kappa_{\kappa}}$ unbounded $\bmod U$ and such that there is no almost one-to-one $g$ with $g \sim f(\bmod U)$. Set

$$
I=\left\{y \subseteq \kappa: \forall \rho<\kappa\left(\left|y \cap f^{-1}(\{\rho\})\right|<\kappa\right)\right\}
$$

LEMMA 3. If $U \in \mu \kappa$ is weakly selective and not regular, then there is a $k$-complete ideal $I \subseteq P(\kappa)-U$ which is dense in $U \bmod (<\kappa)$. 
The next crucial lemma needed in the proof of Lemma 3 is due to A. Kanamori [6].

LEмmA 4 [6]. If $U \in \mu \kappa$ is not regular then there is a least $(\bmod U)$ almost one-to-one function $f \in{ }^{\kappa} \kappa$.

Proof in outline. Suppose that Lemma 4 is false. One can then construct by induction almost one-to-one functions $f_{\alpha} \in{ }^{\kappa}{ }_{\kappa} \quad(\alpha \in \kappa)$ such that for all $\alpha<\beta<\kappa, f_{\beta}<f_{\alpha}(\bmod U)$, and in addition, if $\beta$ is limit, then for all $\alpha<\beta$ and all $\rho \in \kappa, f_{\beta}(\rho) \leqslant f_{\alpha}(\rho)$. We now define sets $x_{\alpha} \in U$ ( $\alpha<\kappa, \alpha$ successor) as follows. If $\alpha=\gamma+n$ where $\gamma<\kappa, \gamma$ is limit and $1 \leqslant n \in \omega$, then

$$
x_{\alpha}=\left\{\rho<\kappa: \forall m \in \omega\left(0 \leqslant m<n \rightarrow f_{\alpha}(\rho)<f_{\gamma+m}(\rho)\right)\right\}
$$

It can be shown that $\left\{x_{\alpha}: \alpha<\kappa, \alpha\right.$ successor $\}$ regularizes $U$.

LEMMA 5. If $U \in \mu \kappa$ is weakly selective and not regular then there is a least $(\bmod U) f \in{ }^{\kappa} \kappa$ unbounded $\bmod U$.

Proof. Immediate from Lemma 4.

Lemma 6. (SCOTt, SEe [7, Theorem 1.8]). Let $U \in \mu \kappa$ and $f \in{ }^{\kappa_{\kappa}}$ be as in Lemma 5. Set $V=\left\{x \subseteq \kappa: f^{-1}(x) \in U\right\}$. Then $V \in \mu \kappa$ and the identity is a least $(\bmod V)$ function unbounded $\bmod V$. Moreover $V$ extends the filter of closed unbounded subsets of $\kappa$.

Sketch of the proof of Lemma 3. Let $U, f$ and $V$ be as in Lemma 6. Let $J$ be the ideal of those $x \subseteq \kappa$ such that $\kappa-x$ contains a closed unbounded subset of $\kappa$. By Lemma $6, J \subseteq P(\kappa)-V$. It is well known that $J$ is $\kappa$-complete and dense $\bmod (<\kappa)$. Set

$$
I=\left\{y \subseteq \kappa: \exists x \in J\left(y \subseteq f^{-1}(x)\right)\right\}
$$

\section{REFERENCES}

1. J. E. Baumgartner, Results and independence proofs in combinatorial set theory, Doctoral Dissertation, Berkeley, Calif., 1970.

2. C. C. Chang and H. J. Keisler, Model theory, North-Holland, Amsterdam, 1973.

3. W. W. Comfort, private communication.

4. W. W. Comfort and N. Hindman, Almost disjoint refining families for uniform ultrafilters, Notices Amer. Math. Soc. 20 (1973), A-644.

5. N. Hindman, On the existence of c-points in $\beta N \mid N$, Proc. Amer. Math. Soc. 21 (1969), 277-280. MR 39 \#922. 
6. A. Kanamori, On irregularity (a xeroxed note).

7. H. J. Keisler and A. Tarski, From accessible to inacessible cardinals. Results holding for all accessible cardinal numbers and the problem of their extension to inaccessible ones, Fund. Math. 53 (1963/64), 225-308. MR 29 \#3385.

8. K. Prikry, Ultrafilters and almost disjoint sets, General Topology and Appl. 4 (1974), 269-282.

DEPARTMENT OF MATHEMATICS, UNIVERSITY OF MINNESOTA, MINNEAPOLIS, MINNESOTA 55455

Current address: School of Mathematics, Institute for Advanced Study, Princeton, New Jersey 08540 